\title{
Mixed Epigastric Axial Pattern Flap Following Cutaneous Hemangiosarcoma Excision in a Male Dog
}

\author{
Jorge Luiz Costa Castro', Vinicius Gonzalez Peres Albernaz', Rafael Ricardo Huppes², \\ Sérgio Santalucia Ramos da Silva ${ }^{3}$, Jair Rodini Engracia Filho ${ }^{4}$ \& Andrigo Barboza de $\mathrm{Nardi}^{5}$
}

\begin{abstract}
Background: Axial pattern flaps use direct cutaneous arteries to enable closure of a skin defect using a large skin segment. Caudal epigastric axial pattern flaps are highly versatile owing to their wide arc of rotation, which includes the preputial area. The presence of abundant loose and elastic skin allows wider flaps. Hemangiosarcoma (HSA) is a malignant neoplasm of endothelial cells with aggressive local behavior and high metastatic rate; the lungs are the most common site of metastasis. This case report aims to describe the use of a caudal epigastric artery-based flap, with preservation of some cranial epigastric artery branches for penis sparing, following resection of a hemangiosarcoma in the right inguinal region of a male dog. Case: An eight-year-old male Pitt Bull dog was referred with a $15 \mathrm{~cm}$, ulcerated hemangiosarcoma in the right inguinal region, near the prepuce. A similar tumor had been removed from the same location two years before. Previous treatment with oral prednisone did not provide satisfactory results. The patient was suffering from intense chronic blood loss and bacterial infection of the tumor tissue. Thoracic radiographs revealed multiple metastatic nodules. Only mild regenerative anemia was found in blood tests. Considerable reductions in tumor size and in the ulcerated surface, decrease in bleeding, and recovery of appetite were observed after 7 days of administration of piroxicam and cephalexin. Surgical resection of the tumor was performed as a palliative treatment with a minimal safety margin of $2 \mathrm{~cm}$, which spared the penis. An epigastric skin flap incorporating the second mammary gland was performed preserving branches of cranial epigastric arteries, and was rotated $160^{\circ}$ to cover the defect created in the right inguinal region and on the body of the penis. A small suture dehiscence, necrosis of the distal border of the flap, seroma, and multidrug-resistant Escherichia coli infection were observed in the postoperative period. Administration of penicillin $\mathrm{V}$ and topical collagenase, placement of a passive drain, and a second surgical procedure to correct the dehiscence were necessary to solve postoperative complications. Piroxicambased metronomic chemotherapy was maintained continuously, as the patient would exhibit worsening of overall condition upon discontinuation of medication. One hundred days after the initial presentation, the animal was euthanized owing to the presence of an osteolytic lesion on the vertebral bodies of L3-L4 and L5-L6, which were suggestive of discosponlylitis or axial bone metastasis.

Discussion: Since lung metastases were present, penectomy and uretrostomy were not considered an option, and a palliative surgical treatment was performed. In this case report, cranial and caudal epigastric flap presented few complications. Caudal epigastric blood vessels are robust and abundant. Distal necrosis was considered a minor complication and was in accordance with others reports. The multidrug resistant infection was considered a nosocomial infection that arised from a long hospital stay. By inhibiting cyclooxygenase, piroxicam decreases angiogenesis, increases apoptosis, and prolongs disease-free intervals with mild toxicity. An excellent response to preoperative administration of piroxicam was crucial when choosing the postoperative protocol. A significant tumor size reduction and a decrease in chronic bleeding associated to improvement in general health were observed with the piroxicam-based treatment. To our knowledge, this is the first report of a mixed (cranial and caudal) epigastric artery skin flap. This type of flap was considered a good option for correction of large defects in the inguinal region and for preservation of the prepuce and penis.
\end{abstract}

Keywords: hemangiosarcoma, cranial, caudal, penis, sparing.

${ }^{1}$ Unidade Hospitalar para Animais de Companhia (UHAC), Pontifícia Universidade Católica do Paraná (PUCPR), Curitiba, PR, Brazil. ${ }^{2}$ Faculdade Ingá, Maringá, PR. ${ }^{3}$ Universidade do Sul de Santa Catarina (UNISUL), Tubarão, SC, Brazil. ${ }^{4}$ Programa de Pós-graduação em Ciência Animal, PUCPR, Curitiba, PR. ${ }^{5}$ Departamento de Clínica e Cirurgia Veterinária, Universidade Estadual Paulista (UNESP), Jaboticabal, SP, Brazil. CORRESPONDENCE: J.L.C. Castro [castrojlc@ gmail.com - Tel.: +55 (41) 3299-4361]. UHAC, Pontifícia Universidade Católica do Paraná (PUCPR). Rod. BR-376, Km 14. Bairro Costeira. CEP 83010-500 São José dos Pinhais, PR, Brazil. 


\section{INTRODUTION}

Axial pattern flaps are a group of skin flaps with at least one direct cutaneous artery and vein emerging from an angiosome. This type of skin flap allows mobilization of larger skin segments when compared to other reconstructive techniques. A 95\% survival area has been described for axial pattern flaps [11]. The caudal superficial epigastric axial pattern flap is a highly versatile flap owing to its wide arc of rotation, and it is used for reconstructions in the caudal abdomen, flank, inguinal region, prepuce, perineum, thigh, and rear limbs [10]. This flap uses three or four mammary glands, which are supplied by caudal superficial epigastric vessels arising from the inguinal canal [10]. The major advantages of this flap are its safety and ample amount of loose, elastic skin, which allows wider flaps than the tissue limited by the mammary glands [10].

Hemangiosarcoma (HSA) is a mesenchymal malignant neoplasm which originates from endothelial cells of blood vessels; it has an aggressive biological behavior and poor prognosis [4]. HSAs can arise in any tissue, but are most commonly found in the spleen, right atrium and auricle, subcutaneous tissue and liver $[2,4]$. HSAs are highly aggressive, both locally and in distant sites, owing to direct access to hematogenous routes and systemic circulation, and the lungs are their most frequent metastatic site [13]. Dermal HSAs are associated with rapid and early onset of metastasis, and short survival time [14].

This paper aims to describe the use of a mixed cranial and caudal superficial epigastric axial pattern flap for closure of a defect in the right inguinal region after a penis-sparing resection of a cutaneous hemangiosarcoma.

\section{CASE}

An eight-year-old neutered male Pitt Bull weighting $30 \mathrm{~kg}$ was referred to the Small Animal Veterinary Hospital with a $15 \mathrm{~cm}$, ulcerated tumor in the right inguinal region, near the body of the penis. The patient had a history of nodule excision near the prepuce two years ago. Eight months after the first surgical procedure, the animal exhibited recurrence of a neoplasm in the same region. Another $10 \mathrm{~cm}$ neoplasm was present on the right abdominal flank. Previous histopathology results were suggestive of hemangiosarcoma and mast cell tumor for the nodules on the right inguinal region and abdominal flank, respectively. The treatment administered to the patient at that time was based on prednisone
$2 \mathrm{mg} / \mathrm{kg}$ PO q24 h, which had minimal effect on tumor size and growth. Intense blood loss and tumor bacterial infection with presence of necrotic tissue were observed.

Enlargement of the bilateral mandibular and right popliteal lymph nodes was detected on physical examination. A $15 \times 10 \mathrm{~cm}$, poorly marginated, firm, ulcerated tumor with irregular surface involving both the epidermis and the dermis was present on the right inguinal region near the body of the penis. A $10 \times 5$ $\mathrm{cm}$, poorly marginated, non-encapsulated tumor with a floating consistency involved both the epidermis and the dermis on the right abdominal flank.

Three-view survey thoracic radiographs revealed multiple nodules of interstitial pattern in the cranial and right middle lobes in both sides. Ultrasound examination detected diffuse hepatic and splenic hypoechoic nodules and an enlarged iliac lymph node $(3.88 \times 1.72 \mathrm{~cm})$. The complete blood count and biochemical profile showed no significant alterations, except for mild regenerative anemia.

Anticipating a future surgical procedure, the animal was medicated with piroxicam ${ }^{1}(0.3 \mathrm{mg} / \mathrm{kg}$ PO q24 $\mathrm{h}$ for fifteen days), omega-3 fatty acids ${ }^{2}$ ( $1 \mathrm{~g} /$ animal PO q24 h until further recommendations), omeprazole ${ }^{3}$ ( $1 \mathrm{mg} / \mathrm{kg}$ PO q24 h for fifteen days), and cephalexin ${ }^{4}$ (30 mg/kg PO q12 h for ten days).

Seven days after commencement of the new treatment protocol, the owner reported a considerable reduction in tumor size, as well as healing of the ulcerated surface and decrease in bleeding. The animal regained its appetite and became more active.

The surgical intervention for neoplasm resection was performed 15 days after referral. The patient was placed in dorsal recumbence. A circular incision with a 2-cm safety margin was made around the tumor, over the ventral midline of the penis, cranially to the prepuce, and on the inner thigh. Penile and neoformed arteries and veins were doubly ligated with 2-0 polyglactin 910 . The enlarged inguinal lymph node was resected with the neoplastic mass. In order to perform a mixed axial rotation flap of cranial and caudal superficial epigastric vessels, an incision on the ventral midline was made starting just cranial to the prepuce and running cranially up to the xiphoid process, with a cranial convex border, bypassing the second mammary gland and returning caudally until the left inguinal region. The flap was undermined bellow the mammary gland tissue and above the external abdominal oblique muscles, taking special 
care to preserve most superficial cranial epigastric branches. The flap length extended from the inguinal mammary gland up to the caudal thoracic gland. After a bridging incision was performed between the tumor defect and the flap bed, the flap was rotated about $160^{\circ}$ to cover the entire wound in the right inguinal region and body of the penis. Some branches of the superficial cranial epigastric artery and vein were ligated in order to allow correct rotation of the flap. The subcutaneous tissue was closed using 3-0 polyglactin 910 suture in a cruciate pattern, and the skin was closed using 3-0 nylon suture in a simple interrupted pattern.

Piroxicam and omeprazol were maintained during the postoperative period, as previously prescribed. Cephalexin ${ }^{4}$ (30 mg/kg PO q12 h for ten days), dipyrone $^{3}$ ( $25 \mathrm{mg} / \mathrm{kg}$ PO q8 $\mathrm{h}$ for 7 days), and tramadol hydrochloride $^{3}$ ( $5 \mathrm{mg} / \mathrm{kg}$ PO q8 $\mathrm{h}$ for seven days) were added to the treatment protocol. The animal remained hospitalized for three days under strict medical care. The initial dressing was changed on the second postoperative day, and then twice daily. Wound cleansing was performed with $0.9 \%$ sterile saline solution.

Ten days after the surgery, the animal was presented in good general condition, without any abnormal clinical sign. A large seroma developed below the flap. Moderate diffuse edema was observed on the right pelvic limb and prepuce. A small caudal/distal portion $(<2 \mathrm{~cm})$ of the flap exhibited a darkish, avascular appearance with purulent discharge. A bacterial culture demonstrated the presence of multidrug-resistant Escherichia coli that was sensitive to chloramphenicol and penicillin. Cephalexin was discontinued and replaced by Penicillin $\mathrm{V}^{5}$ (1,500,000 UI PO q8 h for twelve days), and an ointment with collagenase and chloramphenicol ${ }^{6}$ was added as a topical treatment. Mechanical debridement was necessary 15 days after surgery in order to clear necrotic tissue and allow seroma drainage. All sutures were removed. Seventeen days after the first surgery, an ambulatory intervention was performed to close the open wound on the caudal/distal border of the flap and for placement of a penrose drain in the inguinal region. The wound borders were debrided and sutured in a triangular fashion using 2-0 nylon suture in a simple interrupted pattern. At this moment, antibiotic treatment, including the topical ointment, was discontinued.

Four days after the second procedure, the drain was removed and there was resolution of the seroma. Sutures were removed twenty-one days after the first surgery, when complete healing of the surgical wound was observed and there were no signs of infection. Histopathological analysis confirmed the diagnosis of hemangiosarcoma. Piroxicam-based metronomic chemotherapy $(0.3 \mathrm{mg} / \mathrm{kg}$ PO daily) was maintained continuously because the patient would exhibit worsening of its overall condition, with vomiting, apathy and anorexia, every time medication was discontinued.

After 100 days, the animal exhibited severe cachexia, selective appetite, hyporexia, apathy, prostration, and limited mobility. Neurological examination revealed hyperreflexic patellar, gastrocnemius, sciatic, and cranial tibial reflexes, lack of superficial nociception, decreased response to deep pain perception, as well as meaningful proprioceptive ataxia on both pelvic limbs. Sensation of intense pain was present on the lumbar region. The trunk muscle reflex was absent at the level of L6. Lumbar spine radiography revealed osteolytic and sclerotic lesions of vertebral bodies between L3-L4 and L5-L6. These lesions were suggestive of discospondylitis or axial bone metastasis. In view of the poor prognosis, decreased quality of life, and poor response to palliative treatment, euthanasia was performed. Necropsy exam was not allowed by the owner.

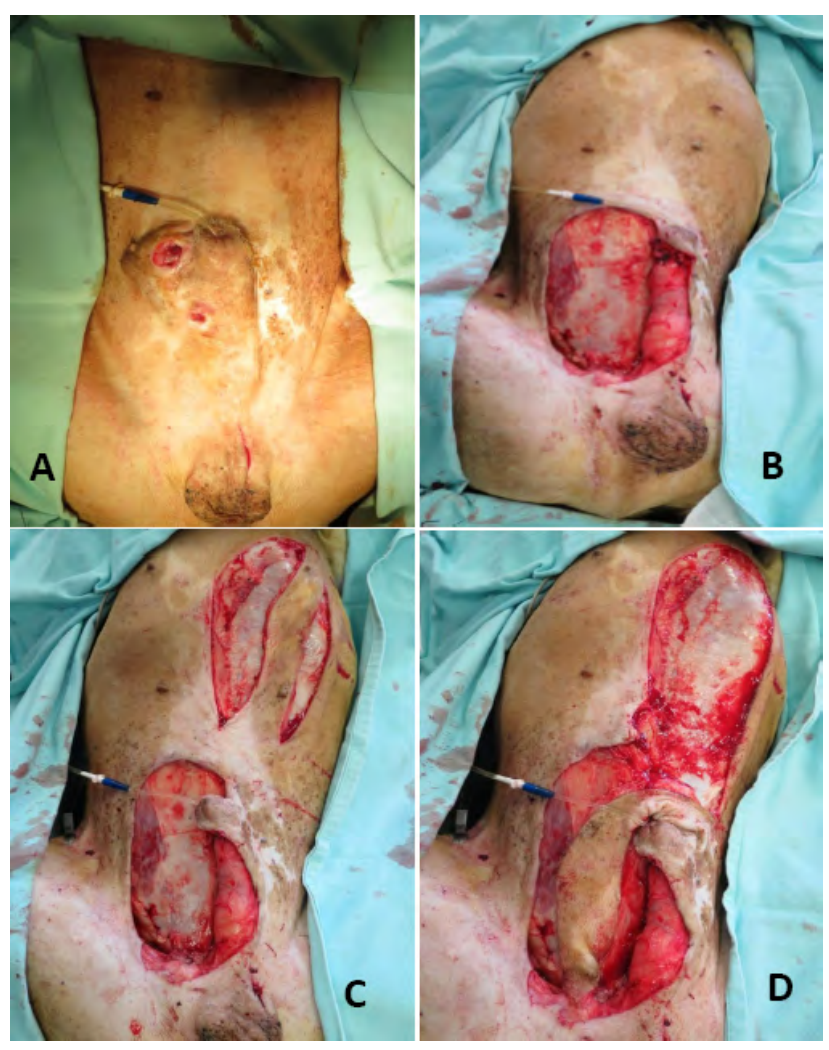

Figure 1. Surgical intervention for resection of an inguinal tumor. (A)- A $15 \mathrm{~cm}$ hemangiosarcoma in the right inguinal region affecting the skin on the body of the penis. (B)- Skin defect after resection. (C)- Mammary gland flap prepared up to the second mammary papilla. (D)- A $160^{\circ}$ flap rotation directed cranially to the prepuce and then caudally to the inguinal region. 


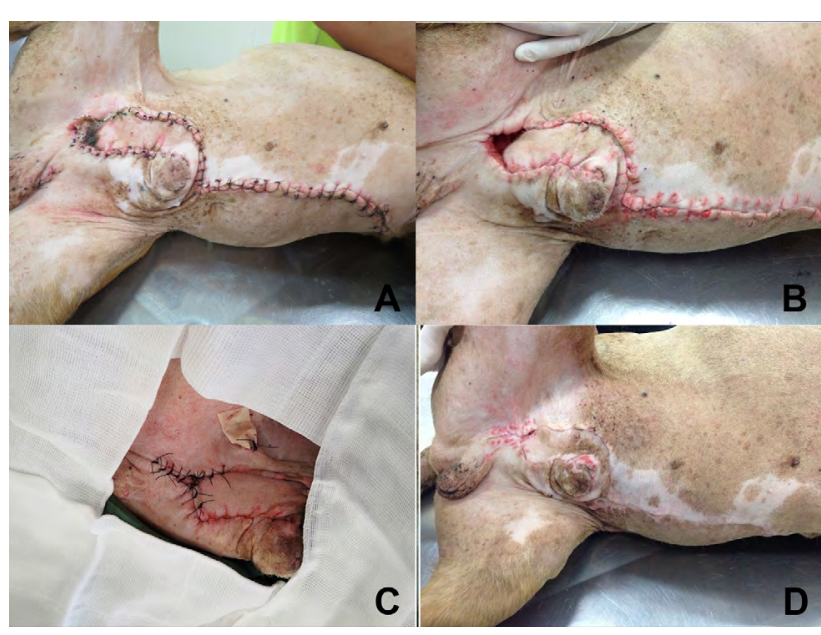

Figure 2. Postoperative appearance of neoplasm resection and superficial mixed cranial and caudal epigastric artery flap. (A)- Skin necrosis of the distal border of the flap seven days after the surgical procedure (B)- Fifteen days after surgery, after debridement and suture removal. (C)- Second surgical intervention for placement of a passive drain and suture of the open wound. (D)- Fifteen days after the surgical closure of the distal border.

\section{DISCUSSION}

A mixed cranial and caudal epigastric axial pattern flap was used in the case described here to allow skin closure while sparing the penis. Penectomy and scrotal uretrostomy were not desired owing to the palliative intent of the surgical intervention. The patient exhibited pulmonary metastasis, so a curative intent surgery was not possible.

Seroma formation is one of the most common complications of a skin flap. A retrospective study on caudal epigastric axial flaps showed a $30 \%$ incidence of seroma formation [1]. In this case report, a seroma was formed in the dead space created between the inguinal muscles and the skin flap about 10 days after surgery, without passive drainage. Necrosis of a small distal flap created a natural defect for the drainage of the seroma.

The distal necrotic area involved less than 5\% of the total flap area, and hence it was considered a minor problem. However, an additional surgical intervention was necessary to close an incisional dehiscence in the necrotic area, and for placement of a penrose drain. After the drain was placed, there was resolution of the seroma. Bruising and flap edema were not observed. A clinical study on caudal superficial epigastric flaps reported a mean flap survival area of $91 \%$. Caudal epigastric blood supply is robust in size and explains the few complications observed [1]. A common cause of skin flap necrosis is the variation of arterial and venous pressure during and/or after anesthesia, which

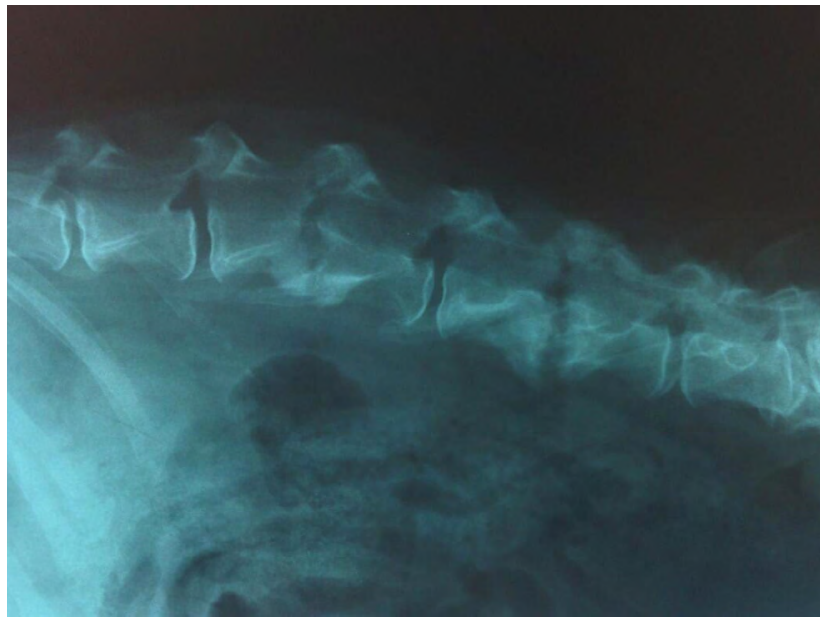

Figure 3. Radiographic image of the lumbar spine showing intense osteolytic and osteoproliferative lesions, and sclerosis on L3-L4 and L5-L6 articular epiphysis, indicative of a metastatic or infectious (discospondylitis) process.

interferes with flap viability $[9,12]$. Other causes of distal border necrosis include microvascular thrombosis and hypoxia due to hypoperfusion [6,7].

The multidrug resistant Escherichia coli culture was considered to be a nosocomial infection that arose from the hospital stay. Sensitivity to penicillin and chloramphenicol allowed association of systemic and topical treatment with fast positive results.

As a cyclooxygenase (COX) inhibitor, piroxicam can elicit significant antineoplastic activity by decreasing angiogenesis and increasing apoptosis in canine transitional cell carcinoma [8]. A retrospective study on incompletely resected soft tissue sarcomas treated with metronomic therapy with cyclophosphamide and piroxicam reported a significant, prolonged disease-free interval and mild toxicity [5]. Piroxicam was chosen as solo therapy owing to the excellent patient response during preoperative administration, providing significant tumor size reduction, decrease in ulcer surface, inflammation, and chronic bleeding, and improvement of general health status. Piroxicam was well tolerated during 100 days of postoperative period until euthanasia. A recent trial of cyclophosphamide with piroxicam on canine oral melanoma xenografted into mice demonstrated potent antiangiogenic effects that reduced tumor growth [3].

To the authors' knowledge, there are no previously published papers reporting caudal epigastric axial pattern flaps with preservation of cranial epigastric branches. 
The use of mixed cranial and caudal superficial epigastric artery flap is a reliable reconstructive technique for inguinal and penile region reconstruction. Using this technique, even a large defect created after a complex neoplasm resection can be closed while preserving the prepuce and penis. The few complications observed in the case reported here did not differ from those described for other skin flaps. The most notable complication observed in the case reported here was necrosis of the distal border with consequent suture dehiscence. Piroxicam-based protocols provide a good response rate with preoperative and postoperative decrease in tumor size, and increase in quality of life.

\author{
MANUFACTURERS \\ ${ }^{1}$ Laboratórios Pfizer. Guarulhos, SP, Brazil. \\ ${ }^{2}$ Sunflower. Araçoiaba da Serra, SP, Brazil. \\ ${ }^{3}$ Medley. Campinas, SP, Brazil. \\ ${ }^{4}$ Nova Química. Barueri, SP, Brazil. \\ ${ }^{5}$ Eurofarma Laboratórios. São Paulo, SP, Brazil. \\ ${ }^{6}$ Cristália. Itapira, SP, Brazil.
}

Declaration of interest. The authors report no conflicts of interest. The authors alone are responsible for the contents and writing of the paper.

\section{REFERENCES}

1 Aper R.L. \& Smeak D.D. 2005. Clinical evaluation of caudal superficial epigastric axial pattern flap reconstruction of skin defects in 10 dogs (1989-2001). Journal of American Animal Hospital Association. 41(3): 185-192.

2 Brown N.O., Patnaik A.K. \& MacEwen E.G. 1985. Canine hemangiosarcoma: retrospective analysis of 104 cases. Journal of American Animal Hospital Association. 186(1): 56-58.

3 Choisunirachon N., Jaroensong T., Yoshida K., Sarki K., Mochizuki M., Nishimura R., Sasaki N. \& Nakagawa T. 2015. Effects of low-dose cyclophosphamide with piroxicam on tumour neovascularization in a canine oral malignant melanoma-xenografted mouse model. Veterinary and Comparative Oncology. 13(4): 424-432.

4 Dervisis N.G., Dominguez P.A., Newman R.G., Cadile C.D. \& Kitchell B.E. 2011. Treatment with DAV for advancedstage hemangiosarcoma in dogs. Journal of American Animal Hospital Association. 47(3): 170-178.

5 Elmslie R.E., Glawe P. \& Dow S.W. 2008. Metronomic therapy with cyclophosphamide and piroxicam effectively delays tumor recurrence in dogs with incompletely resected soft tissue sarcomas. Journal of Veterinary Internal Medicine. 22(6): 1373-1378.

6 Halfacree Z.J., Baines S.J., Lipscomb V.J., Grierson J., Summers B.A. \& Brockman D.J. 2007. Use of a latissimus dorsi myocutaneous flap for one-stage reconstruction of the thoracic wall after en bloc resection of primary rib chondrosarcoma in five dogs. Veterinary Surgery. 36(6): 587-592.

7 Kruse A.L., Luebbers H.T., Grätz K.W. \& Obwegeser J.A. 2010. Factors influencing survival of free-flap in reconstruction for cancer of the head and neck: a literature review. Microsurgery. 30(3): 242-248.

8 Mohammed S.I., Bennett P.F., Craig B.A. Glickman N.W., Mutsaers A.J., Snyder P.W., Widmer W.R., Degortari A.E., Bonney P.L. \& Knapp D.W. 2002. Effect of the cyclooxygenase inhibitor, piroxicam, on tumor response, apoptosis, and angiogenesis in a canine model of human invasive urinary bladder cancer. Cancer Research. 62(2): 356-358.

9 Namndar T., Bartscher T., Stollwerck P.L., Mailänder P. \& Lange T. 2010. Complete Free flap loss due to extensive hemodilution. Microsurgery. 30(3): 214-217.

10 Pavletic M. 2010. Atlas of small animals wound management and reconstructive surgery. 3rd edn. Cambridge: WileyBlackwell, pp.81-430.

11 Pavletic M.M. 1981. Canine axial pattern flaps, using the omocervical, thoracodorsal and deep circumflex iliac direct cutaneous arteries. American Journal of Veterinary Research. 42(3): 391-406.

12 Shridharani S.M., Magarakis M., Manson P.N. \& Rodriguez E.D. 2010. Psychology of plastic and reconstructive surgery: a systematic clinical review. Plastic and Reconstructive Surgery. 126(6): 2243-2251.

13 Thamm D.H. 2007. Hemangiosarcoma. In: Withrow S.J., Vail D.M. \& Page R.L. (Eds) Withrow and MacEwen's Small Animal Clinical Oncology. 5th edn. St. Louis: Saunders, pp.679-688.

14 Ward H., Fox L.E., Calderwood-Mays M.B., Hammer A.S. \& Couto C.G. 1994. Cutaneous Hemangiosarcoma in 25 Dogs: A retrospective study. Journal of Veterinary Internal Medicine. 8(5): 345-348. 